\title{
A systematic review of the effect of individualized risk communication strategies on screening uptake and its psychological predictors: the role of psychology theory
}

\author{
Kathryn Bould, Blanaid Daly, Stephen Dunne, Suzanne Scott and Koula Asimakopoulou \\ Division of Population and Patient Health, King's College London, UK
}

\begin{abstract}
People might be more likely to attend for health screening if they are told their individual risk of an illness. The way this risk of ill-health is communicated might have an effect on screening uptake or its psychological proxies. It is possible that the format, presentation, and details of the information as well as the complexity of an intervention and use of psychological theory to inform the intervention may impact the effectiveness of individual risk communication. This systematic review collates, analyses and synthesizes the evidence for effectiveness of these aspects of individual risk communication. The synthesis indicated that written, individualized risk scores or categories are effective at supporting screening uptake and its psychological proxies. Complex, or theory-based interventions, surprisingly, are no more effective than simpler or atheoretical interventions.
\end{abstract}

\section{Introduction}

A significant proportion of mortality from the leading causes of death in the industrialized world is due to modifiable behavior patterns (Connor and Norman, 2005). The psychological predictors of such health behavior patterns (e.g. attendance for screening) have been researched extensively and several social cognition models

Correspondence: Kathryn Bould, Division of Population and Patient Health, King's College London, Floor 18, Tower Wing, Guy's Hospital, London SE1 9RW, UK.

Tel.: +44.207.848.5145.

E- mail: kathryn.bould@kcl.ac.uk

Key words: Systematic review, Risk communication, Screening, Psychological predictors.

Contributions: the authors contributed equally.

Conflict of interest: the authors declare no potential conflict of interest.

Received for publication: 7 September 2016.

Accepted for publication: 16 September 2016.

This work is licensed under a Creative Commons AttributionNonCommercial 4.0 International License (CC BY-NC 4.0).

(C) Copyright K. Bould et al., 2016

Licensee PAGEPress, Italy

Health Psychology Research 2016; 4:6157

doi:10.4081/hpr.2016.6157
(SCMs) have been put forward e.g. Health Belief Model, Protection Motivation Theory (Connor and Norman, 2005). Psychological theory such as SCMs often focuses on predicting health behavior through the notion of threat or risk. For instance, people consider, amongst other psychological factors, their risk for a particular condition before choosing to engage in a health behavior. SCMs seeking to predict adherence to health behaviours suggest that people consider their risk for a particular condition, whether it is expressed as their perceived risk, perceived susceptibility, or their perceived severity for developing a disease, before engaging in any health behaviour. Therefore, targeting an individual's understanding of their own risk for a particular disease may lead to behavior change, such as screening uptake by enhancing behavior-change proxies such as intentions to undertake screening, knowledge and attitudes etc. Therefore, theory-driven research would suggest that risk is an important concept to examine, so that an individual's understanding of their own risk for a particular disease or condition can be targeted to inform the decision to undergo screening.

There is a diverse range of tests and screening programs that can be used to identify those who are at risk of developing various diseases or conditions. Many of these tests aim to differentiate between those who probably have a disease, and those who probably do not, or aim to highlight a risk of disease (Edwards et al., 2006). Screening programs often provide information about population risks of developing a disease to inform decision-making regarding screening, or they aim to motivate people to attend for testing in order to maximize screening uptake (Edwards et al., 2013). However, not all studies in the field use theoretical models to design their interventions, and not all screening programs offer individualized risk information. A popular way to target and improve screening uptake is to provide information that is personally relevant; often known as individualized risk. Recent systematic reviews have reported evidence of enhanced informed decision-making in those who received individualized risk information with, surprisingly, highly detailed risk communication, predicting lower screening uptake (Edwards et al., 2006; 2013). Proxies of screening uptake were considered in the review, but the theoretical background of the reviewed studies was not investigated. Another systematic review specifically on cardiovascular risk communication (Waldron, van der Weijden, Ludt, Gallacher and Elwyn, 2011) found that presenting patients with individualized risk in percentages or frequencies, using graphical representation and short timeframes, was best for eliciting behavior change. However, this review focused on cardiovascular risk only and did not attempt to explore whether theory-based interventions were more effective than atheoretical work in eliciting behavior change or whether certain presentation details such as who delivered the risk information and whether it was simple of complex details that were given were best. If the purpose of risk communication is to lead to behavior change such as screening uptake, then theoretical work, such as social cognition models designed to inform behavior change interventions may be useful in designing risk communication interventions. 
Given the importance of risk communication in healthcare (Moher, Liberati, Tetzlaff, and Altman, 2009), it is important to identify the most effective strategies for risk communication at an individual level. Understanding however of how best to present and discuss risks and benefits of health care in general, and screening in particular is still limited. The previous systematic reviews though informative, have not sought to explain why less detailed risk information (Edwards et al., 2013) presented in graphs, scores or categories (Waldron et al., 2011) is effective in maximizing screening uptake. If the purpose of risk communication is to lead to behavior change, then psychology theory such as, for example, social cognition models designed to inform behavior change interventions, may be useful in understanding what combinations of individualized risk communication (IRC) components work best. The purpose of this systematic review was to consider the effects of IRC on screening uptake or its psychosocial proxies, as proposed by psychological theory. If IRC interventions explicitly informed by theory are found to lead to better outcomes than a-theoretical interventions, the use of theory in designing IRC interventions would be justified. This systematic review aimed to address the following questions: Are theory-driven risk IRC interventions to increase screening uptake or its psychological proxies more successful than a-theoretical interventions? What are the most effective IRC strategies to increase screening uptake or its psychological proxies?

\section{Materials and Methods}

The Preferred Reporting Items for Systematic Reviews and MetaAnalyses (Moher et al., 2009) guidelines were adhered to throughout.

\section{Eligibility criteria}

Randomized controlled trials (RCTs), defined as conveying probabilistic individualized information about a disease, personal risk factors, susceptibility, or the risks of taking a test were sought. Studies with people facing real life decisions and analogue studies were included. The screening methods had to involve investigations performed by health professionals. Studies were included where interventions provided information on individualized risk, categorization of risk status such as, high/low or a discussion of personal risk factors. IRC could be delivered via oral, written, video, or via electronic media formats. RCTs providing information on individualized risk compared with another IRC, a control arm receiving usual care or generalized risk information, were included. Screening uptake was considered the primary outcome. Studies were also considered for inclusion if there was a psychological predictor of behavior (e.g. fear, intention). These psychological predictors of (proxies) were considered secondary outcomes.

Papers were excluded if they were not written in English or published before $31^{\text {st }}$ December 2005, as they were included in the most up-to-date Cochrane review on risk communication at the time of our search strategy (Edwards et al., 2006).

\section{Study identification}

Comprehensive search strategies were developed and conducted in July 2012. An electronic database search (EMBASE, MEDLINE, PsycINF0, Web of Science and PubMed) was supplemented by a manual search of relevant journals, author names and reference lists of included papers and past systematic reviews.

Eligibility assessment was performed independently by two reviewers (KB and KA). Disagreements between reviewers were resolved in discussion. Titles and abstracts of articles were examined for relevance and duplicates were removed. Full texts of articles were further screened for eligibility, and those not meeting the inclusion criteria were excluded.

\section{Data extraction}

Using a standardized data extraction sheet data were extracted by two independent reviewers (KB and $\mathrm{KA}$ ) regarding Participants, Intervention, Comparators, Outcomes (PICO). The Cochrane's Risk of Bias tool was used by two reviewers working independently (Higgins and Green, 2011). No studies were excluded from the review on the basis of their risk of bias.

Observed and total numbers from studies with dichotomous outcomes such as screening uptake, and means/ mean change and standard deviations for studies with continuous outcomes, such as knowledge and worry, were noted in order to arrive at the narrative conclusion.

\section{Synthesis of results}

Due to the heterogeneous outcome variables and the nature of the designs, a meta-analysis was deemed unworthy. Thus, a narrative synthesis on all interventions is reported.

\section{Results}

\section{Study selection}

The PRISMA flow chart shows the stages of the study selection process (Figure 1). Of the final twenty-one relevant articles, three studies were each reported in two articles, therefore in total, eighteen studies were included. Study characteristics are outlined in Supplementary Table S1.

\section{Participants and settings}

The studies had a total of 22,557 participants. There were four times more women $(\mathrm{N}=16,064)$ than men $(\mathrm{N}=4716)$. The majority of studies recruited healthy participants, whilst seven studies recruited participants thought or known to be at higher risk than average for the
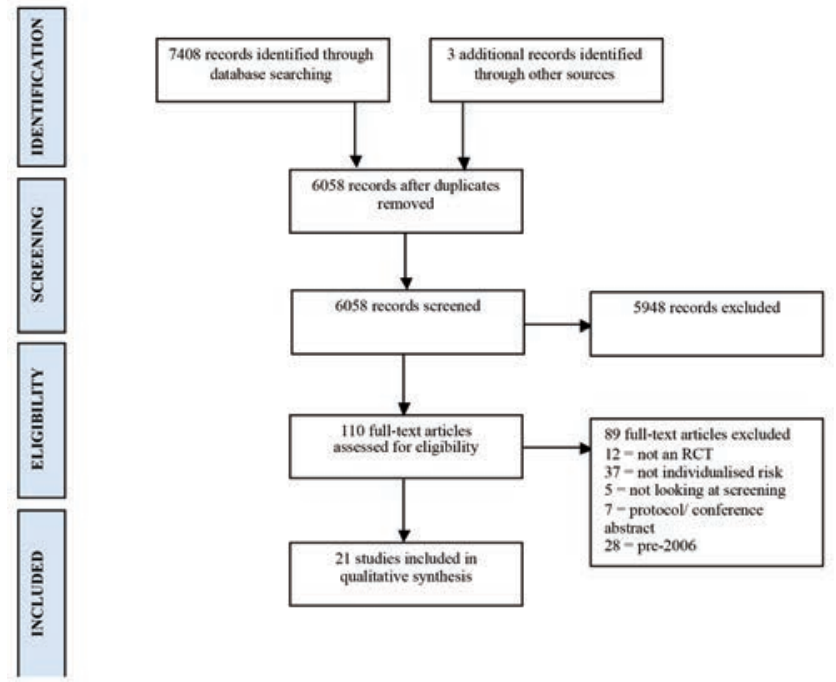

Figure 1. CONSORT diagram of studies through the review process. 
screened condition (Bodurtha et al., 2009; D. J. Bowen, Burke, Culver, Press, and Crystal, 2006; Glanz, Steffen, and Taglialatela, 2007; Glenn et al., 2011; Lipkus and Klein, 2006; Manne et al., 2009; Rawl et al., 2012). Participants' age ranged from 18-74 years. Whilst some studies did not report the study setting, the majority of interventions were delivered in primary care $(\mathrm{N}=7)$ or participants' own environment $(\mathrm{N}=5)$. The majority of studies were USA-based $(\mathrm{N}=14)$.

\section{Interventions}

Seven of the eighteen studies used psychological theory to inform their intervention. Supplementary Table $\mathrm{S} 2$ shows which interventions had a theoretical basis.

The Health Belief Model (Bloom, Stewart, Chang, and You, 2006; Bodurtha et al., 2009; Manne et al., 2009; Rawl et al., 2012; Vernon et al., 2008), the Transtheoretical Model (Bodurtha et al., 2009; Manne et al., 2009; Vernon et al., 2008), Self-Regulatory Theory (Bodurtha et al., 2009; D. J. Bowen et al., 2011), Precaution Adoption Process Model (Glanz et al., 2007), and Dual Process Theory (Manne et al., 2009) were used. One study based their intervention on several psychological theoretical concepts (Deborah J. Bowen and Powers, 2010), whilst one other used the Risk Reappraisal Hypothesis (Glenn et al., 2011).

Interventions addressed several diseases. The most common $(\mathrm{N}=7$ studies) was screening for colorectal cancer, followed by screening for breast cancer or its gene $(\mathrm{N}=5)$. Four of the eighteen RCTs had two IRC intervention arms (D. J. Bowen et al., 2006; Helmes, Culver, and Bowen, 2006; Lipkus and Klein, 2006; Manne et al., 2009) leading to a total of twenty-two IRC interventions. Within studies, intervention arms differed in risk presentation format. (Supplementary Table S2). Of the intervention arms studied, $N=7$ presented an IRC score. Six interventions presented a categorization of risk level such as above/below average. Whilst $\mathrm{N}=4$ studies listed or discussed personal risk factors, $\mathrm{N}=5$ interventions were unclear as to the type of risk presented.

Risk presentation format varied largely across intervention arms. Of the twenty-two intervention arms, two communicated risk verbally in person, whilst two supplemented this with a written communication. One intervention was computer-aided, whilst a further three interventions used supplemented the computer aid with a printout. Seven interventions gave participants written risk information only, two interventions gave written risk information and then reviewed the information verbally in person, two interventions provided written risk information followed by a telephone discussion, two interventions communicated the individualized risk through a specialized website, and finally, one intervention communicated risk solely over the telephone.

Interventions where the risk information was presented in person or over the telephone were delivered by a variety of people such as counselors and staff specifically trained to deliver interventions. Detailed characteristics of the intervention deliverers such as training and monitoring the delivery of the risk information for treatment fidelity purposes were largely omitted. This meant that it was difficult to see if the risk information delivered in this format was delivered in a comparable manner to all participants in the study. Consequently, this meant that any kind of replication of the intervention without this detail would be problematic.

All studies provided IRC in conjunction with other information, e.g. educational material. The intervention material differed in complexity. A total of thirteen studies provided participants with some form of educational material regarding a disease/screening test (Allen et al., 2010; Bodurtha et al., 2009; D. J. Bowen et al., 2006; Deborah J. Bowen and Powers, 2010; D. J. Bowen et al., 2011; Glenn et al., 2011; Harari et al., 2008; Helmes et al., 2006; Lipkus and Klein, 2006; Rawl et al., 2012; Schroy et al., 2011; Schroy et al., 2012; Steckelberg, Hulfenhaus,
Haastert, and Muhlhauser, 2011; Yuksel, Majumdar, Biggs, and Tsuyuki, 2010; Yuksel, Tsuyuki, and Majumdar, 2012). In two studies educational information was provided alongside a decision-aid tool (Allen et al., 2010; Schroy et al., 2011; Schroy et al., 2012).Six studies discussed theoretical constructs such as barriers, benefits and self-efficacy, alongside the individualized risk information (Bloom et al., 2006; Bodurtha et al., 2009; Glanz et al., 2007; Glenn et al., 2011; Manne et al., 2009; Vernon et al., 2008). Two studies provided personalized screening recommendations based on risk level (Glanz et al., 2007; Rawl et al., 2012). Two studies provided follow-up phone-calls (Deborah J. Bowen and Powers, 2010; Glanz et al., 2007), whilst six studies tailored the additional material to the individual (D. J. Bowen et al., 2011; Glenn et al., 2011; Lipkus and Klein, 2006; Manne et al., 2009; Rawl et al., 2012; Vernon et al., 2008). Five studies presented a relative risk (Allen et al., 2010; Deborah J. Bowen and Powers, 2010; Helmes et al., 2006; Lipkus and Klein, 2006; Steckelberg et al., 2011), one presented natural frequencies (Steckelberg et al., 2011), and $\mathrm{N}=3$ presented lifetime risk (Bloom et al., 2006; Bodurtha et al., 2009; Deborah J. Bowen and Powers, 2010). Two studies reported the individualised risk numerically (D. J. Bowen et al., 2011; Helmes et al., 2006), whilst $\mathrm{N}=5$ presented risk graphically (Allen et al., 2010; Deborah J. Bowen and Powers, 2010; D. J. Bowen et al., 2011; Helmes et al., 2006; Vernon et al., 2008). One study (two articles) used color-coded categories to present risk (Rubinstein et al., 2011; Ruffin et al., 2011).

Overall, reporting of intervention details was average to poor; some studies lacked details of the risk format and intervention (Glenn et al., 2011; Manne et al., 2009; Steckelberg et al., 2011; Vernon et al., 2008).

\section{Outcome and follow-up}

See Supplementary Table S1 for the outcome measures for each study. Seven studies (Bloom et al., 2006; Bodurtha et al., 2009; D. J. Bowen et al., 2011; Glanz et al., 2007; Manne et al., 2009; Rawl et al., 2012; Vernon et al., 2008) reported outcomes explicitly predicted by social-cognition models. $\mathrm{N}=1$ study did not explicitly use one theory to design the intervention but based it on several theoretical concepts (Deborah J. Bowen and Powers, 2010). The remaining ten studies did not explicitly use theory to inform outcomes, but their outcomes were nevertheless encompassed by psychological constructs seen in social cognition models. All twenty-one studies reported baseline $v s$. postintervention information, although follow-up timing varied among studies. No two studies measured the same outcomes. Eleven studies measured actual screening uptake, while $\mathrm{N}=4$ measured screening intentions, and $\mathrm{N}=1$ measured actual combined with planned screening uptake. Secondary outcomes varied even more. Perceived risk was measured in eight studies; other common outcome measures included knowledge and worry.

\section{Results of individual studies}

Most ( $N=14 / 18)$ studies reported some degree of positive findings; IRC-based interventions had a significant effect on screening uptake $(\mathrm{N}=2)$, its psychological predictors $(\mathrm{N}=6)$, or both $(\mathrm{N}=6)$. One study found no effects on either outcome (Bloom et al., 2006), whilst $\mathrm{N}=3$ found no effect on either screening uptake or its psychological predictors but saw a significant increase in related health behaviors such as fruit and vegetable consumption (Rubinstein et al., 2011; Ruffin et al., 2011), exercise (Bodurtha et al., 2009; Harari et al., 2008; Rubinstein et al., 2011; Ruffin et al., 2011) and vaccination uptake (Harari et al., 2008). In some interventions the use of IRC had some effect on participants' screening behavior or psychological predictors of screening uptake compared to generalized risk information or a control arm. Screening uptake was significantly higher in the intervention compared to control arms in $\mathrm{N}=8$ studies, and $\mathrm{N}=12$ studies reported sig- 
nificant secondary effects such as higher screening intentions, more accurate risk perceptions, less worry etc. Therefore, it appears that IRC may have a positive impact on both screening uptake and its psychological proxies. Seven studies (Bloom et al., 2006; Bodurtha et al., 2009; D. J. Bowen et al., 2011; Glanz et al., 2007; Manne et al., 2009; Rawl et al., 2012; Vernon et al., 2008) used social cognition models to inform the intervention, $\mathrm{N}=1$ used several psychological concepts (Deborah J. Bowen and Powers, 2010), whilst ten did not. These ten studies, measured outcomes related to behavior-change psychology theory such as knowledge, perceived risk, and worry. There were no differences in outcomes between interventions informed by theory and a-theoretical ones. For example, increases in knowledge, perceived risk and screening uptake, reductions in worry and interest in genetic testing, were seen both in studies that used theory to inform their intervention, and studies that did not (Supplementary Table S1).

\section{Type of risk presented}

Of the five studies that communicated a risk score, $\mathrm{N}=2$ reported an increase in screening uptake (Deborah J. Bowen and Powers, 2010; D. J. Bowen et al., 2011), while $\mathrm{N}=3$ reported better psychological outcomes (e.g. worry) (Deborah J. Bowen and Powers, 2010; D. J. Bowen et al., 2011; Helmes et al., 2006). Of the six studies that presented an individualized risk category, $\mathrm{N}=2$ reported an increase of screening uptake (Glanz et al., 2007; Yuksel et al., 2010; Yuksel et al., 2012) whilst $\mathrm{N}=3$ reported better psychological outcomes e.g. knowledge (Allen et al., 2010; Glanz et al., 2007; Schroy et al., 2011; Schroy et al., 2012). Of the three studies (four intervention arms) that presented a list of personal risk factors, screening uptake did not increase in any, however, $\mathrm{N}=2$ reported better psychological outcomes such as fewer barriers to screening (Lipkus and Klein, 2006; Rawl et al., 2012). It is possible that studies using a risk score or categorizing risk may be more successful at increasing uptake of screening tests compared to simply listing personal risk factors. However, this conclusion is made with caution as some studies reporting an increase in screening uptake, lacked detail about the type of risk presented (Glenn et al., 2011; Manne et al., 2009; Steckelberg et al., 2011; Vernon et al., 2008).

\section{Disease or condition and screening test}

Interventions successfully increased screening uptake in a variety of diseases. However, the two interventions providing IRC about a variety of diseases or conditions, were less successful at enhancing screening uptake compared to interventions focusing on one condition (Supplementary Table S1).

\section{Type of participants}

Seven studies recruited people thought to be at greater risk of developing a particular disease/condition. Of these, $\mathrm{N}=3$ reported increased screening uptake with IRC (Glanz et al., 2007; Glenn et al., 2011; Manne et al., 2009), whilst $\mathrm{N}=6$ reported improved psychological outcomes (D. J. Bowen et al., 2006; Glanz et al., 2007; Glenn et al., 2011; Lipkus and Klein, 2006; Manne et al., 2009; Rawl et al., 2012). Eleven studies recruited average risk participants; of these, $\mathrm{N}=4$ reported enhanced screening uptake with IRC (Deborah J. Bowen and Powers, 2010; D. J. Bowen et al., 2011; Vernon et al., 2008; Yuksel et al., 2010; Yuksel et al., 2012), whilst $\mathrm{N}=6$ reported improved psychological outcomes (Deborah J. Bowen and Powers, 2010; D. J. Bowen et al., 2011; Helmes et al., 2006; Schroy et al., 2011; Schroy et al., 2012; Steckelberg et al., 2011). Given the heterogeneity in risk presentation format, there was no evidence of better screening uptake with high rather than average-risk participants.

\section{Risk information format}

Notwithstanding heterogeneity in formats, there was more support for some risk information formats than others. Seven intervention arms presented risk information in writing, of which $\mathrm{N}=4$ reported an increase in screening uptake (Deborah J. Bowen and Powers, 2010; Glenn et al., 2011; Manne et al., 2009; Vernon et al., 2008), and $\mathrm{N}=5$ reported improved psychological outcomes (D. J. Bowen et al., 2006; Deborah J. Bowen and Powers, 2010; Glenn et al., 2011; Manne et al., 2009; Steckelberg et al., 2011). Two intervention arms provided written risk information first, followed by a discussion of the risk in person, reporting better psychological outcomes but no effect on screening uptake (Lipkus and Klein, 2006). Similarly, $\mathrm{N}=1$ study presenting individualised risk information on a computer, improved several psychological proxies (knowledge and screening intention), but not screening uptake (Schroy et al., 2011; Schroy et al., 2012). Three interventions using a computer first, followed by a printout providing written risk information had no effect on screening uptake, although $\mathrm{N}=2$ reported better psychological outcomes (Allen et al., 2010; Rawl et al., 2012). Of the two intervention arms that communicated risk verbally in person, $\mathrm{N}=1$ reported an increase in screening uptake (Yuksel et al., 2010; Yuksel et al., 2012), and the other reported better psychological outcomes (D. J. Bowen et al., 2006). Two interventions communicated the risk verbally in person then provided the risk in writing. Both studies reported better psychological outcomes (Glanz et al., 2007; Helmes et al., 2006), whilst $\mathrm{N}=1$ reported an increase in screening uptake also (Glanz et al., 2007). Both interventions that provided written risk information followed by a telephone discussion reported better psychological outcomes (Helmes et al., 2006; Manne et al., 2009), whilst $\mathrm{N}=1$ also reported an increase in screening uptake (Manne et al., 2009). Two interventions entailed IRC through a specialized website, and whilst $\mathrm{N}=1$ reported no effects (Rubinstein et al., 2011; Ruffin et al., 2011) the other reported better screening uptake and psychological outcomes (D. J. Bowen et al., 2011). Finally, one intervention communicated risk solely over the telephone, with no improvement in any outcomes (Bodurtha et al., 2009). Overall, all IRC information formats, except that of telephone risk communication, had some form of beneficial outcome, mainly a significant effect on psychological outcomes. It appears that where IRC information is presented in writing, or in a combination with another format (such as communication verbally in person), screening uptake is enhanced. This may be due to the fact that receiving written IRC information either alone, or in combination with another form of communication, was the most frequently used presentation format with at least half of all intervention participants receiving some sort of written IRC.

\section{Details of risk presentation}

Thirteen interventions provided educational information, and all except two reported better outcomes compared to controls (Bodurtha et al., 2009; Harari et al., 2008). Both interventions using a decision-aid reported better psychological outcomes compared to the control arm, and the six studies which discussed theoretical model constructs reported increased screening uptake or psychological outcomes. Two studies using a check-in follow-up call also reported enhanced screening uptake and better psychological outcomes (Deborah J. Bowen and Powers, 2010; Glanz et al., 2007). Finally, tailored interventions presenting individualized risk information reported some level of better outcome in terms of increased screening uptake, better psychological outcomes, or both (D. J. Bowen et al., 2011; Glenn et al., 2011; Lipkus and Klein, 2006; Manne et al., 2009; Rawl et al., 2012; Vernon et al., 2008). In studies presenting relative risk, $\mathrm{N}=5$ studies reported positive effects on screening or psychological outcomes, where $\mathrm{N}=1$ study reported positive screening uptake and better psychological outcomes 
(Deborah J. Bowen and Powers, 2010). Better psychological outcomes were seen in a study presenting natural frequencies (Steckelberg et al., 2011). Therefore, the evidence is inconclusive as to which intervention strategy results in the best overall outcome for increasing screening uptake, as there is very little by way of intervention presentation detail that is not effective.

\section{Simple versus complex IRC interventions}

Four of the eighteen studies compared two IRC arms, varying in complexity, with a control arm (D. J. Bowen et al., 2006; Helmes et al., 2006; Lipkus and Klein, 2006; Manne et al., 2009). In one study, both intervention arms received the same IRC information in writing but one was supplemented with one-to-one counseling, whereas the other received group counseling. Screening uptake improved equally in both counseling arms (D. J. Bowen et al., 2006). In another study two intervention arms received written IRC information, but whilst one arm received theirs during in-person counseling, the other was mailed it and received telephone counseling. There was no difference in psychological outcomes between the two intervention arms, and both had better psychological outcomes than the controls (Helmes et al., 2006). In a further study both intervention arms received individualized absolute risk information, however one arm also received relative risk. Although the more complex intervention increased screening intentions compared to the simple intervention arm, there was no difference in actual screening uptake between interventions (Lipkus and Klein, 2006). Finally, in the fourth study, both intervention arms received IRC information in tailored print, however one also received telephone counseling; the more complex intervention of the two. Again, both intervention arms enhanced screening uptake equally, compared to controls (Manne et al., 2009). Therefore it was concluded that more complex interventions do not have an additive effect on outcomes; they are just as effective as simpler IRC interventions.

\section{Quality of studies}

Overall, individualised risk communication interventions were poorly reported, and the presentation detail was not described in order to answer the review question and identify a full effective strategy for communicating individualised risk information. Further to this, there were several problems noted for studies included overall. Firstly, not all studies measured screening uptake, but those studies that did were largely based upon self-reported receipt of a screening test. Within studies measuring screening uptake, only a few studies confirmed receipt of a screening test by consulting physicians or medical records. Secondly, follow up times varied massively between studies from immediately postintervention up to two years. Ismail and colleagues suggest that a lack of an appropriate post-test measure can reduce clarity of conclusions; they further suggest that it is useful to have longer term measures, to see whether the impact of the intervention does stay with the participant (Ismail, Winkley, and Rabe-Hesketh, 2004). This is especially important when assessing follow-up screening uptake as many screening tests are only carried out annually or even every two or three years, meaning that shorter follow-up times might not be sensitive enough to detect changes in screening adherence. Conversely, all scales and measures used in the studies were validated and checked for reliability and validity, which increases the confidence we can place in the results obtained. Additionally, all 18 of the studies were RCTs, and had an adequate control group. Overall, there was a high level of risk information presentation detail omitted in the included studies which leads to difficulty in proposing one most effective IRC strategy.

\section{Risk of bias}

Assessment through the Cochrane Risk of Bias tool indicated that studies were of variable but acceptable quality. The majority were ade- quate for random sequence generation, but only few reported adequate concealment of allocation to groups. Most studies were unclear about assessor blinding in outcome measures although the majority used patient-reported or objective measures for key outcomes such as assessing test uptake from registers. Adequate incomplete outcome data handling was seen across studies. Some selective reporting across was present, and the methodological reporting across the majority of the studies was poor; in a lot of cases, it was unclear exactly how the IRC was delivered.

\section{Discussion}

Notwithstanding the moderate quality of reviewed studies and the heterogeneity in methods and outcomes, there is support for a wide range of IRC strategies to increase screening uptake and its psychological proxies. Psychology theory has been used in designing IRC interventions to increase screening uptake or its psychological proxies and such studies are as effective in improving screening or its psychological proxies, as a-theoretical studies. Our first research question was, Are theory-driven risk IRC interventions to increase screening uptake or its psychological proxies more successful than a-theoretical interventions? We found no evidence to suggest that the few interventions that explicitly used theory to inform IRC were more successful at enhancing behavioral outcomes than atheoretical studies. This could be because theory is truly unhelpful in predicting outcomes, or because intervention fidelity was poor in the reported interventions, or possibly because of improper use of behavior change techniques. Interestingly, all of the atheoretical studies measured outcomes that are supported by theory (such as e.g. attitudes) albeit the absence of theory to actually drive the design of the intervention per se. We found some evidence to answer our second research question, What are the most effective IRC strategies to increase screening uptake or its psychological proxies? There was some evidence that that more detailed types of risk presented, such as risk score or category, the slightly more successful the intervention at increasing screening uptake and its psychological predictors compared to simply listing personal risk factors. However, there were interventions that were successful at increasing screening uptake and its psychological outcomes that did not report on the type of risk presented so this result is to be taken with caution (Manne et al., 2009; Steckelberg et al., 2011; Vernon et al., 2008). In line with Edwards, our results suggest that average and high risk participants do as well as each other in risk interventions whilst simple interventions are as effective as complex ones (Edwards et al., 2013). Equally, providing individualized risk about one rather than several diseases may be better for outcomes. The role of a limited capacity attention processor may explain this finding; where the risk of just one disease is presented, individuals are more likely to be able to focus and process the information in order to make a decision about attending for screening (Kahneman, 1973). The majority of successful studies presented IRC information in writing, or in combination with another format (e.g. such as verbally in person) so there seems to be a substantial amount of support of its effect on screening uptake. This may be because participants preferred to have the information to hand to review again, or to take in at their own pace.

This systematic review builds on the findings from Edwards et al, and compliments their latest review reporting that most risk communication work is on cancer, although a few other conditions have recently been examined (Edwards et al., 2013). Since their review, there have been a few other clinical topic areas examined, such as prostate cancer and osteoporosis and mixed clinical topic conditions, where studies have examined risk of developing several diseases or conditions together. Secondly, like Edwards, we have found there to be more IRC 
risk scores and categories of risk being utilised rather than simply listing personal risk factors, and these appeared to impact actual screening uptake and its psychological proxies. In particular, although several presentation methods may be successful at increasing outcomes, presenting the risk in writing or in combination with another format, such as verbally in person, was a popular and effective choice. Finally, the few IRC interventions informed by psychological theory were no more effective at increasing screening uptake than a-theoretical ones, although most a-theoretical interventions reported on outcomes encompassed by theory.

\section{Strengths and limitations of this review}

A relatively small number of studies heavily based on cancer risk with a possible selection bias due to the exclusion of papers pre-2006 and non-English language studies has informed this review. Notwithstanding some well-reported studies, overall, intervention format and delivery details were reported inconsistently and the presentation detail was not described in order to answer the review question and identify a full effective strategy for communicating individualized risk information. Further, inconsistency in intervention outcomes, a lot of which relied on self-report was also present. Albeit a few additions, the variety of clinical topics is still quite narrow as originally stated in Edwards review in 2006, with the majority of studies producing results in the field of colorectal cancer and breast cancer (Edwards et al., 2006). Additionally, there is a possible selection bias in the current review with the exclusion of papers pre-2006 and of non-English language studies. Further to this, there were several problems noted for studies included overall. Firstly, not all studies measured screening uptake, but those studies that did were largely based upon self-reported receipt of a screening test. Within studies measuring screening uptake, only a few studies confirmed receipt of a screening test by consulting physicians or medical records. Secondly, follow up times varied massively between studies from immediately post-intervention up to two years. A lack of an appropriate post-test measure can reduce clarity of conclusions; therefore it is useful to have longer term measures, to see whether the impact of the intervention does stay with the participant. This is especially important when assessing follow-up screening uptake as many screening tests are only carried out annually or even every two or three years, meaning that shorter follow-up times might not be sensitive enough to detect changes in screening adherence. However, the strength of the results lies in the fact that the studies have been gathered from systematic searches of several key databases and contact with key authors in the field, and represent a narrative synthesis of the most recent literature. Whilst the risk of bias tool was used to assess the quality of the studies included in the review, a full quality assessment of the studies to assess the strength of the evidence could have been conducted using CASP checklists for instance (CASP, 2014).

\section{Practical implications}

Current evidence suggests that discussing health risks using simple risk scores or categories and providing this information in writing, may be successful in supporting people to consider undertaking health screening. There are of course cost implications to any intervention. What is promising is that the more complex interventions (which presumably, by their very nature, are more costly) do not appear to be any more effective than simpler interventions. However it would be useful to formally assess the cost-effectiveness of the reviewed interventions in order to inform the feasibility of implementation of integrating IRC into screening programs. Insufficient information is provided in the existing studies to perform such an evaluation. Future risk communication intervention studies should aim to include this information so that the cost effectiveness of the intervention can be assessed.

\section{Conclusions}

This review has found evidence to suggest that individualized risk communication is more effective than generalized or no risk information at supporting screening uptake and its psychological proxies. Whilst presenting individualized risk information in written format and expressing the risk as an individualized score or category may be more effective at increasing screening uptake, complex, or theory-driven interventions (e.g. counseling or education), are no more effective than simpler or a-theoretical interventions. It has also demonstrated the need for more well-reported studies in individualized risk communication, focusing on a wider range of clinical field. A wide range of outcomes have been measured too, but there has been little consistency in risk presentation.

\section{References}

Allen, J.D., Othus, M.K.D., Hart, A., Tom, L., Li, Y., Berry, D., and Bowen, D. (2010). A Randomized Trial of a Computer-Tailored Decision Aid to Improve Prostate Cancer Screening Decisions: Results from the Take the Wheel Trial. Cancer Epidemiology Biomarkers and Prevention, 19, 2172-2186.

Bloom, J.R., Stewart, S.L., Chang, S., and You, M. (2006). Effects of a telephone counseling intervention on sisters of young women with breast cancer. Preventive Medicine, 43, 379-384.

Bodurtha, J., Quillin, J.M., Tracy, K. A., Borzelleca, J., McClish, D., Wilson, D.B., .. . Bowen, D. (2009). Mammography Screening after Risk-Tailored Messages: The Women Improving Screening through Education and Risk Assessment (WISER) Randomized, Controlled Trial. Journal of Womens Health, 18, 41-47.

Bowen, D.J., Burke, W., Culver, J.O., Press, N., and Crystal, S. (2006). Effects of counseling Ashkenazi Jewish women about breast cancer risk. Cultural diversity and ethnic minority psychology, 12, 4556.

Bowen, D.J., and Powers, D. (2010). Effects of a mail and telephone intervention on breast health behaviors. Health Education and Behavior, 37, 479-489.

Bowen, D.J., Robbins, R., Bush, N., Meischke, H., Ludwig, A., and Wooldridge, J. (2011). Effects of a Web-based intervention on women's breast health behaviors. Translational Behavioral Medicine, 1, 155-164.

CASP. (2014). CASP Checklists. Retrieved from http://www.caspuk.net/casp-tools-checklists

Connor, M., and Norman, P. (2005). Predicting Health Behaviour: A Social Cognition Approach. In M. Connor and P. Norman (Eds.), Predicting Health Behaviour (pp. 1-27). England: Open University Press.

Edwards, A.G., Evans, R., Dundon, J., Haigh, S., Hood, K., and Elwyn, G. J. (2006). Personalised risk communication for informed decision making about taking screening tests. Cochrane database of systematic reviews (Online), 4, CD001865.

Edwards, A.G., Naik, G., Ahmed, H., Elwyn, G.J., Pickles, T., Hood, K., and Playle, R. (2013). Personalised risk communication for informed decision making about taking screening tests. Cochrane Database Syst Rev, Cd001865.

Glanz, K., Steffen, A.D., and Taglialatela, L.A. (2007). Effects of colon cancer risk counseling for first-degree relatives. Cancer Epidemiology, Biomarkers and Prevention, 16, 1485-1491.

Glenn, B.A., Herrmann, A.K., Crespi, C.M., Mojica, C.M., Chang, L.C., Maxwell, A.E., and Bastani, R. (2011). Changes in risk perceptions 
in relation to self-reported colorectal cancer screening among firstdegree relatives of colorectal cancer cases enrolled in a randomized trial. Health Psychology, 30, 481-491.

Harari, D., Iliffe, S., Kharicha, K., Egger, M., Gillmann, G., von RentelnKruse, W., ... Stuck, A. (2008). Promotion of health in older people: a randomised controlled trial of health risk appraisal in British general practice. Age and Ageing, 37, 565-571.

Helmes, A.W., Culver, J.0., and Bowen, D.J. (2006). Results of a randomized study of telephone versus in-person breast cancer risk counseling. Patient Education and Counseling, 64, 96-103.

Higgins, J., and Green, S. (2011). Cochrane Handbook for Systematic Reviews of Interventions (Vol. Version 5.1.0 [updated March 2011]): The Cochrane Collaboration.

Ismail, K., Winkley, K., and Rabe-Hesketh, S. (2004). Systematic review and meta-analysis of randomised controlled trials of psychological interventions to improve glycaemic control in patients with type 2 diabetes. Lancet, 363, 1589-1597.

Kahneman, D. (1973). Attention and Effort. Englewood Cliffs NJ: Prentice Hall.

Lipkus, I., and Klein, W. (2006). Effects of communicating social comparison information on risk perceptions for colorectal cancer. Journal of Health Communication, 11, 391-407.

Manne, S.L., Coups, E.J., Markowitz, A., Meropol, N.J., Haller, D., Jacobsen, P.B., . . . Winkel, G. (2009). A randomized trial of generic versus tailored interventions to increase colorectal cancer screening among intermediate risk siblings. Annals of Behavioral Medicine, 37, 207-217.

Moher, D., Liberati, A., Tetzlaff, J., and Altman, D.G. (2009). Preferred reporting items for systematic reviews and meta-analyses: the PRISMA statement. BMJ, 339, b2535.

Rawl, S.M., Skinner, C.S., Perkins, S.M., Springston, J., Wang, H.L., Russell, K.M., . . Champion, V.L. (2012). Computer-delivered tailored intervention improves colon cancer screening knowledge and health beliefs of African-Americans. Health Education Research, $27,868-885$.

Rubinstein, W. S., Acheson, L. S., O'Neill, S. M., Ruffin, M. T., Wang, C., Beaumont, J. L., . . . Family Healthware Impact Trial, F. H. I. (2011).
Clinical utility of family history for cancer screening and referral in primary care: A report from the Family Healthware Impact Trial. Genetics in Medicine, 13, 956-965.

Ruffin, M.T.I.V., Nease, D. E., Jr., Sen, A., Pace, W.D., Wang, C., Acheson, L.S., .. Gramling, R. (2011). Effect of preventive messages tailored to family history on health behaviors: The Family Healthware Impact Trial. Annals of Family Medicine, 9, 3-11.

Schroy, P.C., Emmons, K., Peters, E., Glick, J.T., Robinson, P.A., Lydotes, M.A., . . Heeren, T.C. (2011). The Impact of a Novel ComputerBased Decision Aid on Shared Decision Making for Colorectal Cancer Screening: A Randomized Trial. Medical Decision Making, 31, 93-107.

Schroy, P.C., Emmons, K.M., Peters, E., Glick, J.T., Robinson, P.A., Lydotes, M.A., . . . Heeren, T.C. (2012). Aid-assisted decision making and colorectal cancer screening: a randomized controlled trial. American Journal of Preventive Medicine, 43, 573-583.

Steckelberg, A., Hulfenhaus, C., Haastert, B., and Muhlhauser, I. (2011). Effect of evidence based risk information on "informed choice" in colorectal cancer screening: randomised controlled trial. BMJ, 342, d3193.

Vernon, S.W., del Junco, D.J., Tiro, J.A., Coan, S.P., Perz, C.A., Bastian, L.A., ... DiClemente, C. (2008). Promoting regular mammography screening II. Results from a randomized controlled trial in US women veterans. Journal of the National Cancer Institute, 100, 347-358.

Waldron, C.A., van der Weijden, T., Ludt, S., Gallacher, J., and Elwyn, G. (2011). What are effective strategies to communicate cardiovascular risk information to patients? A systematic review. Patient Education and Counseling, 82, 169-181.

Yuksel, N., Majumdar, S.R., Biggs, C., and Tsuyuki, R.T. (2010). Community pharmacist-initiated screening program for osteoporosis: randomized controlled trial. Osteoporosis International, 21(3), 391-398. doi:10.1007/s00198-009-0977-z

Yuksel, N., Tsuyuki, R.T., and Majumdar, S.R. (2012). Predictors of Bone Mineral Density Testing in Patients at High Risk of Osteoporosis: Secondary Analyses From the OSTEOPHARM Randomized Trial. Journal of Clinical Densitometry, 15, 61-66. 